TAIWANESE JOURNAL OF MATHEMATICS

Vol. 13, No. 6A, pp. 1837-1846, December 2009

This paper is available online at http://www.tjm.nsysu.edu.tw/

\title{
GAP FUNCTIONS FOR NONSMOOTH EQUILIBRIUM PROBLEMS
}

\author{
Marco Castellani and Massimo Pappalardo
}

\begin{abstract}
We consider equilibrium problems $(E P)$ with directionally differentiable (not necessarily $\mathcal{C}^{1}$ ) bifunctions which are convex with respect to the second variable and we use a gap function approach to solve them. In the first part of the paper we establish a condition under which any stationary point of the gap function solves $(E P)$ and we propose a solution method which uses descent directions of the gap function. In the final section we study the problem when this condition is not satisfied. In this case we use a family of gap functions depending on a parameter $\alpha$ which allows us to overcome the trouble due to the lack of a descent direction.
\end{abstract}

\section{INTRODUCTION}

Different kinds of competitive situations (see for example $[2,5]$ and references therein) can be formulated via general equilibrium model of this type

$$
\text { find } \bar{x} \in C \text { s.t. } f(\bar{x}, y) \geq 0, \quad \forall y \in C,
$$

where $C \subseteq \mathbb{R}^{n}$ is a compact convex set and the equilibrium bifunction $f \in \mathcal{A}$ where $\mathcal{A}=\left\{f: \mathbb{R}^{n} \times \mathbb{R}^{n} \rightarrow \mathbb{R}: f(\cdot, y)\right.$ is directionally differentiable for all $y \in C$,

$$
f(x, \cdot) \text { is convex for all } x \in C, f(z, z)=0 \text { for all } z \in C\}
$$

Recently an increasing effort has been made to develop algorithms for computing equilibrium solutions. Some of them are based on the fact that $(E P)$ can be reformulated as an optimization problem via gap functions. This has been proposed also in $[1,3,6,7,8]$. In this paper we focus on $(E P)$ in which the bifunction $f$ is only directionally differentiable and not $\mathcal{C}^{1}$. The scheme proposed follows the same line of [1].

Received August 7, 2009.

2000 Mathematics Subject Classification: 90C30, 49M37.

Key words and phrases: Equilibrium problems, Gap functions. 
The following notation will be used in the paper. If $f \in \mathcal{A}$ we denote the directional derivative of $f(\cdot, y)$ at $x$ along the direction $u$ by

$$
D_{x} f(x, y ; u)=\lim _{t \rightarrow 0^{+}} \frac{f(x+t u, y)-f(x, y)}{t} .
$$

Analogously $D_{y} f(x, y ; v)$ indicates the directional derivative of $f(x, \cdot)$ at $y$ along the direction $v$.

The following function

$$
\varphi(x)=-\min _{y \in C} f(x, y)
$$

was introduced in [7]. We prove, for the sake of completeness, that it is a gap function for $(E P)$.

Theorem 1.1. Let $f \in \mathcal{A}$ be given; then

(i) $\varphi(x) \geq 0$ for all $x \in C$;

(ii) $\bar{x} \in C$ verifies $\varphi(\bar{x})=0$ if and only if $\bar{x}$ solves $(E P)$.

Proof. For all $x \in C$ we have

$$
\varphi(x)=-\min _{y \in C} f(x, y) \geq-f(x, x)=0
$$

proving statement $(i)$. If $0=\varphi(\bar{x})=-\min _{y \in C} f(\bar{x}, y)$ then $\bar{x} \in C$ solves $(E P)$; the converse is trivial since $f(z, z)=0$ for all $z \in C$ and this proves (ii).

Next sections are devoted to seek for descent directions of the gap function $\varphi$ in order to minimize it.

\section{Strictly Convex and Strictly D-Monotone Equilibrium Problems}

In this section we suppose that $f \in \mathcal{A}$ is strictly convex with respect to $y$, for all $x \in C$. This assumption, together with the compactness of $C$, implies the existence of the unique minimizer $y(x) \in C$ such that

$$
\varphi(x)=-f(x, y(x)) .
$$

The next result of Danskin [4] permits to compute the directional derivative of $\varphi$. 
Theorem 2.1. Let $f \in \mathcal{A}$ be strictly convex with respect to $y$; the function $x \mapsto y(x)$ is continuous and $\varphi$ is directionally differentiable with

$$
D \varphi(x ; v)=-D_{x} f(x, y(x) ; v)
$$

for all $x \in C$ and $v \in \mathbb{R}^{n}$.

Since $f(z, z)=0$, it is easy to show that the solution set of $(E P)$ coincides with the set of the fixed points of the function $x \mapsto y(x)$, i.e. $\bar{x} \in C$ is a solution of $(E P)$ if and only if $\bar{x}=y(\bar{x})$. When $\bar{x} \neq y(\bar{x})$, in order to establish whether $y(\bar{x})-\bar{x}$ is a descent direction for $\varphi$, additional assumptions on $f$ are usually assumed in literature (see for instance $[3,6,8]$ ). We will use the following.

Definition 2.1. A bifunction $g \in \mathcal{A}$ is called strictly $D$-monotone on $C$ if

$$
D_{x} g(x, y ; y-x)>D_{y} g(x, y ; x-y), \quad \forall x, y \in C \text { with } x \neq y .
$$

If $g \in \mathcal{A}$ is continuously differentiable, the concept of strict $D$-monotonicity collapses with the concept of strict $\nabla$-monotonicity introduced in [1]. It is easy to prove that the concept of strict $D$-monotonicity is not related to the classical concept of strict monotonicity. Several nice properties hold.

Theorem 2.2. Suppose that $f \in \mathcal{A}$ is strictly convex with respect to $y$ and strictly D-monotone on $C$, then

$$
D \varphi(x ; y(x)-x)<0, \quad \forall x \in C \text { with } x \neq y(x) .
$$

Proof. From Theorem 2.1 and the strict $D$-monotonicity of $f$ we deduce

$$
D \varphi(x ; y(x)-x)=-D_{x} f(x, y(x) ; y(x)-x)<-D_{y} f(x, y(x) ; x-y(x)) ;
$$

since $y(x)$ is a global minimum of $f(x, \cdot)$ over $C$, the first order necessary optimality condition implies

$$
D_{y} f(x, y(x) ; x-y(x)) \geq 0
$$

that concludes the proof.

Strict $D$-monotonicity guarantees also the following "stationarity property" for the reformulation of $(E P)$ as optimization problem through $\varphi$.

Theorem 2.3. Suppose that $f \in \mathcal{A}$ is strictly convex with respect to $y$ and strictly $D$-monotone on $C$; if $\bar{x}$ is a stationary point of $\varphi$ over $C$, i.e.

$$
D \varphi(\bar{x} ; y-\bar{x}) \geq 0, \quad \forall y \in C,
$$

then $\bar{x}$ solves $(E P)$. 
Proof. By contradiction, suppose that $\bar{x}$ does not solve $(E P)$ and hence $y(\bar{x}) \neq$ $\bar{x}$. Since $y(\bar{x})$ is a global minimum for the function $f(\bar{x}, \cdot)$ we deduce

$$
D_{y} f(\bar{x}, y(\bar{x}) ; \bar{x}-y(\bar{x})) \geq 0 \text {. }
$$

Moreover, from (4) valued at $y(\bar{x})$ and Theorem 2.1 we obtain

$$
\left.D_{x} f(\bar{x}, y(\bar{x})) ; y(\bar{x})-\bar{x}\right) \leq 0 .
$$

But (5) and (6) contradict the assumption of strict $D$-monotonicity of $f$.

The results proved in Theorem 2.2 and Theorem 2.3 give us a solution method for solving $(E P)$. In fact, we have a descent direction (Theorem 2.2), a stopping rule (Theorem 2.3), and we can propose an exact linesearch rule to find the stepsize. The iterative sequence of the solution method is given

$$
x^{k+1}=x^{k}+t_{k} d^{k}
$$

where $d^{k}=y\left(x^{k}\right)-x^{k}$ and $t_{k} \in[0,1]$ minimizes $\theta(t)=\varphi\left(x^{k}+t d^{k}\right)$ over $[0,1]$. Since $d^{k}$ depends with continuity upon $x^{k}$, convergence to a stationary point of $\varphi$ is achieved via Zangwill's Theorem.

\section{Convex and Strictly $D$-Monotone Equilibrium Problems}

When $f(x, \cdot)$ is not strictly convex, we have not the uniqueness of the minimum point $y(x)$. For this reason we consider a continuously differentiable bifunction $h: \mathbb{R}^{n} \times \mathbb{R}^{n} \rightarrow \mathbb{R}$ such that

(a) $h(x, y) \geq 0$ for all $x, y \in C$ and $h(z, z)=0$ for all $z \in C$,

(b) $h(x, \cdot)$ is strictly convex for all $x \in C$.

As immediate consequence of (a) and (b) we have that $\nabla_{y} h(z, z)=0$, for all $z \in C$.

We define the bifunction $F=f+h$ and we introduce the following auxiliary equilibrium problem

$$
\text { find } \bar{x} \in C \text { s.t. } F(\bar{x}, y) \geq 0, \quad \forall y \in C \text {. }
$$

The next result shows the equivalence between $(E P)$ and $(A E P)$.

Theorem 3.1. The point $\bar{x}$ solves $(E P)$ if and only if $\bar{x}$ solves $(A E P)$. 
Proof. Trivially, every solution of $(E P)$ solves $(A E P)$. Vice versa let $\bar{x}$ be a solution of $(A E P)$ and suppose, by contradiction, there exists $\bar{y} \in C$ such that $f(\bar{x}, \bar{y})<0$. Since $h$ is continuously differentiable, $h(\bar{x}, \bar{x})=0$ and $\nabla_{y} h(\bar{x}, \bar{x})=0$, we have

$$
h(\bar{x}, \bar{x}+t(\bar{y}-\bar{x}))=\|\bar{x}-\bar{y}\| t \omega(t), \quad \forall t \in(0,1]
$$

where $\omega(t)$ tends to 0 for $t \rightarrow 0^{+}$. Therefore, from the convexity of $f(\bar{x}, \cdot)$ and since $f(\bar{x}, \bar{x})=0$, we deduce

$$
\begin{aligned}
0 & \leq F(\bar{x}, \bar{x}+t(\bar{y}-\bar{x})) \\
& =f(\bar{x}, \bar{x}+t(\bar{y}-\bar{x}))+h(\bar{x}, \bar{x}+t(\bar{y}-\bar{x})) \\
& \leq(1-t) f(\bar{x}, \bar{x})+t f(\bar{x}, \bar{y})+\|\bar{x}-\bar{y}\| t \omega(t) \\
& =t[f(\bar{x}, \bar{y})+\|\bar{x}-\bar{y}\| \omega(t)] .
\end{aligned}
$$

Since $f(\bar{x}, \bar{y})+\|\bar{x}-\bar{y}\| \omega(t)<0$ for $t$ sufficiently small, we achieve the contradiction.

Theorem 3.1 allows us to apply the results of the previous section to the gap function associated to the bifunction $F$ and for this we need the strict $D$-monotonicity of $F$.

Definition 3.1. A bifunction $g \in \mathcal{A}$ is called $D$-monotone on $C$ if

$$
D_{x} g(x, y ; y-x) \geq D_{y} g(x, y ; x-y), \quad \forall x, y \in C .
$$

If $g$ is continuously differentiable the concept of $D$-monotonicity collapses with the concept of $\nabla$-monotonicity defined in [1], i.e.

$$
\left\langle\nabla_{x} g(x, y)+\nabla_{y} g(x, y), y-x\right\rangle \geq 0, \quad \forall x, y \in C .
$$

All the usually used bifunctions $h$ as, for instance, the square of the euclidean norm $h(x, y)=\|x-y\|^{2}$ are $D$-monotone but not strictly $D$-monotone.

It is easy to show that if $f$ is strictly $D$-monotone and $h$ is $D$-monotone then $F$ is strictly $D$-monotone. So we can adapt to this case the solution method presented at the end of Section 2.

\section{A Larger Class of Equilibrium Problems}

Since strict $D$-monotonicity is not always verified by the bifunction $f$, we now analyse the case when $f$ doesn't satisfy this condition but it is only $D$-monotone. In this case (even if $f(x, \cdot)$ is strictly convex) Theorem 2.2 can not be applied. In fact, 
if we substitute the assumption of strict $D$-monotonicity with $D$-monotonicity, it is possible to show that $y(x)-x$ is not always a descent direction (see [1, Example 2.5]). For this reason we substitute the auxiliary bifunction $F$ with a family of bifunctions $F_{\alpha}=f+\alpha h$, with $\alpha>0$ and we denote by $\varphi_{\alpha}$ the associated gap function. We will show that the parameter $\alpha$ allows us to overcome the troubles due to the lack of the "stationarity property". Anyway, we will require the following additional assumption on $f$

$$
f(x, y)+D_{x} f(x, y ; y-x) \geq 0, \quad \forall x, y \in C .
$$

It is possible to prove that condition (8) is stronger than $D$-monotonicity.

Theorem 4.1. If the bifunction $f \in \mathcal{A}$ satisfies (8) then it is $D$-monotone.

Proof. From the convexity of $f(x, \cdot)$ we have

$$
0=f(x, x) \geq f(x, y)+D_{y} f(x, y ; x-y), \quad \forall x, y \in C ;
$$

therefore, the above inequality and (8) guarantee

$$
\begin{aligned}
& D_{x} f(x, y ; y-x)-D_{y} f(x, y ; x-y) \\
= & {\left[f(x, y)+D_{x} f(x, y ; y-x)\right]-\left[f(x, y)+D_{y} f(x, y ; x-y)\right] \geq 0 }
\end{aligned}
$$

for all $x, y \in C$ and thus $f$ is $D$-monotone.

Some examples presented in [1] show that no relationship exists between condition (8) and the strict $D$-monotonicity and, moreover, that the stationarity property is not guaranteed for a fixed gap function $\varphi_{\alpha}$. When condition (8) holds, we can overcome the trouble of finding a descent direction by eventually modifying the parameter $\alpha$ and therefore by changing the considered gap function.

Theorem 4.2. Suppose $f \in \mathcal{A}$ satisfies (8) and assume that

$$
\begin{aligned}
& \lim _{y^{\prime} \rightarrow y} \lim _{t \rightarrow 0^{+}} \frac{f\left(x+t\left(y^{\prime}-x\right), y^{\prime}\right)-f\left(x, y^{\prime}\right)}{t} \\
= & \lim _{t \rightarrow 0^{+}} \lim _{y^{\prime} \rightarrow y} \frac{f\left(x+t\left(y^{\prime}-x\right), y^{\prime}\right)-f\left(x, y^{\prime}\right)}{t},
\end{aligned}
$$

for all $x, y \in C$. If $x \in C$ is not a solution of $(E P)$, then there exists $\bar{\alpha}$ such that $y_{\alpha}(x)-x$ is a descent direction at $x$ for all positive $\alpha \leq \bar{\alpha}$.

Proof. Suppose, by contradiction, there exists a sequence $\left\{\alpha_{k}\right\} \downarrow 0$ such that

$$
D \varphi_{\alpha_{k}}\left(x ; y_{\alpha_{k}}(x)-x\right) \geq 0
$$


Since $C$ is compact, we can suppose that the sequence $\left\{y_{\alpha_{k}}(x)\right\}$ converges to $y \in C$. By assumption

$$
f_{\alpha_{k}}\left(x, y_{\alpha_{k}}(x)\right)=-\varphi_{\alpha_{k}}(x)<0
$$

and therefore, since $f(x, \cdot)$ is continuous, taking the limit for $k \rightarrow \infty$, we deduce that

$$
f(x, y)=\lim _{k \rightarrow \infty} f_{\alpha_{k}}\left(x, y_{\alpha_{k}}(x)\right) \leq 0 .
$$

On the other hand $y_{\alpha_{k}}$ minimizes $f_{\alpha_{k}}(x, \cdot)$ over $C$, then

$$
D_{y} f_{\alpha_{k}}\left(x, y_{\alpha_{k}}(x) ; x-y_{\alpha_{k}}(x)\right) \geq 0 \text {. }
$$

Let $a>D_{y} f(x, y ; x-y)$ then there exists $t_{0} \in(0,1)$ such that $y+t(x-y) \in C$ and

$$
\frac{f(x, y+t(x-y))-f(x, y)}{t}<a
$$

for all $t \in\left(0, t_{0}\right)$. Moreover $f\left(x, y_{\alpha_{k}}(x)+t\left(x-y_{\alpha_{k}}(x)\right)\right)$ tends to $f(x, y+t(x-y))$ and $f\left(x, y_{\alpha_{k}}(x)\right)$ tends to $f(x, y)$ for $k \rightarrow \infty$. Hence, for $k$ sufficiently large, we have

$$
\frac{f\left(x, y_{\alpha_{k}}(x)+t\left(x-y_{\alpha_{k}}(x)\right)\right)-f\left(x, y_{\alpha_{k}}(x)\right)}{t}<a .
$$

Since

$$
D_{y} f\left(x, y_{\alpha_{k}}(x) ; x-y_{\alpha_{k}}(x)\right) \leq \frac{f\left(x, y_{\alpha_{k}}(x)+t\left(x-y_{\alpha_{k}}(x)\right)\right)-f\left(x, y_{\alpha_{k}}(x)\right)}{t}
$$

it follows that

$$
\limsup _{k \rightarrow \infty} D_{y} f\left(x, y_{\alpha_{k}}(x) ; x-y_{\alpha_{k}}(x)\right) \leq a
$$

This is true for any $a>D_{y} f(x, y ; x-y)$ and then

$$
\begin{aligned}
D_{y} f(x, y ; x-y) & \geq \limsup _{k \rightarrow \infty} D_{y} f\left(x, y_{\alpha_{k}}(x) ; x-y_{\alpha_{k}}(x)\right) \\
& =\limsup _{k \rightarrow \infty} D_{y} f_{\alpha_{k}}\left(x, y_{\alpha_{k}}(x) ; x-y_{\alpha_{k}}(x)\right) \geq 0 .
\end{aligned}
$$

Therefore, from Theorem 4.1, we deduce that

$$
D_{x} f(x, y ; y-x) \geq 0 .
$$

Condition (10) can be written

$$
D_{x} f_{\alpha_{k}}\left(x, y_{\alpha_{k}}(x) ; y_{\alpha_{k}}(x)-x\right) \leq 0
$$

and taking the limit for $k \rightarrow \infty$ and using condition (9) we have the converse inequality

$$
D_{x} f(x, y ; y-x) \leq 0
$$


and therefore

$$
D_{x} f(x, y ; y-x)=0 .
$$

Since condition (8) holds, we have $f(x, y) \geq 0$ and therefore we deduce $f(x, y)=0$. Moreover $f_{\alpha_{k}}\left(x, y_{\alpha_{k}}(x)\right) \leq f_{\alpha_{k}}\left(x, y^{\prime}\right)$ for all $y^{\prime} \in C$, hence, taking the limit again,

$$
0=f(x, y) \leq f\left(x, y^{\prime}\right), \quad \forall y^{\prime} \in C .
$$

This implies that $x$ solves $(E P)$ in contradiction with the assumption.

When $f$ is continuously differentiable, condition (9) is trivially satisfied. The above result provides the key idea for the solution method for $D$-monotone bifunctions: decrease the value of $\alpha$ whenever $y_{\alpha}(x)-x$ isn't any longer a descent direction for $\varphi_{\alpha}$ and apply the scheme presented in Section 2 .

Nevertheless, in order to device a new kind of solution method more efficient from the computational point of view, we have to implement an Armijo-type rule for the stepsize. If we adopt this kind of rule, we need the following theorem.

Theorem 4.3. Suppose that $f \in \mathcal{A}$ satisfies condition (8) and $h$ is $\nabla$-monotone then

$$
\begin{aligned}
& D \varphi_{\alpha}\left(x ; y_{\alpha}(x)-x\right) \\
\leq & f\left(x, y_{\alpha}(x)\right)-\alpha\left\langle\nabla_{x} h\left(x, y_{\alpha}(x)\right), y_{\alpha}(x)-x\right\rangle \leq 0, \quad \forall x \in C .
\end{aligned}
$$

Proof. The first inequality in (11) descends immediately from condition (8) since

$$
\begin{aligned}
D \varphi_{\alpha}\left(x ; y_{\alpha}(x)-x\right) & =-D_{x} f_{\alpha}\left(x, y_{\alpha}(x) ; y_{\alpha}(x)-x\right) \\
& =-D_{x} f\left(x, y_{\alpha}(x) ; y_{\alpha}(x)-x\right)-\alpha\left\langle\nabla_{x} h\left(x, y_{\alpha}(x)\right), y_{\alpha}(x)-x\right\rangle \\
& \leq f\left(x, y_{\alpha}(x)\right)-\alpha\left\langle\nabla_{x} h\left(x, y_{\alpha}(x)\right), y_{\alpha}(x)-x\right\rangle .
\end{aligned}
$$

For the second inequality in (11), since $y_{\alpha}(x)$ is a global minimum for $f_{\alpha}(x, \cdot)$, the first order necessary optimality condition implies

$$
\begin{aligned}
& 0 \leq D_{y} f_{\alpha}\left(x, y_{\alpha}(x) ; x-y_{\alpha}(x)\right) \\
= & D_{y} f\left(x, y_{\alpha}(x) ; x-y_{\alpha}(x)\right)+\alpha\left\langle\nabla_{y} h\left(x, y_{\alpha}(x)\right), x-y_{\alpha}(x)\right\rangle .
\end{aligned}
$$

Moreover $h$ is $\nabla$-monotone then

$$
\begin{aligned}
& D_{y} f\left(x, y_{\alpha}(x) ; x-y_{\alpha}(x)\right) \\
\geq & \alpha\left\langle\nabla_{y} h\left(x, y_{\alpha}(x)\right), y_{\alpha}(x)-x\right\rangle \geq-\alpha\left\langle\nabla_{x} h\left(x, y_{\alpha}(x)\right), y_{\alpha}(x)-x\right\rangle .
\end{aligned}
$$


From the convexity of $f(x, \cdot)$ we obtain

$$
0=f(x, x) \geq f\left(x, y_{\alpha}(x)\right)+D_{y} f\left(x, y_{\alpha}(x) ; x-y_{\alpha}(x)\right),
$$

and hence

$$
D_{y} f\left(x, y_{\alpha}(x) ; x-y_{\alpha}(x)\right) \leq-f\left(x, y_{\alpha}(x)\right) .
$$

Comparing (12) and (13) we deduce the required second inequality.

Theorem 4.3 gives us an upper estimate of the directional derivative of the gap function. This is a fundamental result in order to obtain a globally convergent algorithm as we have seen in the continuously differentiable case [1]. In fact exploiting (11) we can force the gap function to have a decrease which is large enough. In particular the direction will be accepted when the inequality

$$
-\varphi_{\alpha}(x)-\alpha\left(\left\langle\nabla_{x} h\left(x, y_{\alpha}(x)-x\right\rangle+h\left(x, y_{\alpha}(x)\right)\right)<-\frac{1}{2} \varphi_{\alpha}(x)\right.
$$

holds. Naturally we can work decreasing the parameter $\alpha$. In fact, if $x$ is not a solution of $(E P)$ condition (14) ensures that the direction is a descent direction (see Theorem 4.3).

\section{REFERENCES}

1. G. Bigi, M. Castellani and M. Pappalardo, A new solution method for equilibrium problems, Optimization Methods and Software, to appear. DOI: $10.1080 / 10556780902855620$.

2. E. Blum and W. Oettli, From optimization and variational inequalities to equilibrium problems, The Mathematics Student, 63 (1993), 1-23.

3. O. Chadli, I. V. Konnov and J. C. Yao, Descent methods for equilibrium problems in a Banach space, Computers and Mathematics with Applications, 48 (2004), 609-616.

4. J. M. Danskin, The theory of min-max with applications, SIAM Journal on Applied Mathematics, 14 (1966), 641-664.

5. A. Iusem and W. Sosa, New existence results for equilibrium problems, Nonlinear Analisys, 52 (2003), 621-635.

6. I. V. Konnov and M. S. S. Ali, Descent methods for monotone equilibrium problems in Banach spaces, Journal of Computational and Applied Mathematics, 188 (2006), 165-179.

7. G. Mastroeni, On auxiliary principle for equilibrium problems, in: Equilibrium problems and variational models, P. Daniele, F. Giannessi and A. Maugeri (eds.), Kluwer Academic Publishers, Dordrecht, 2003, pp. 289-298. 
8. G. Mastroeni, Gap functions for equilibrium problems, Journal of Global Optimization, 27 (2003), 411-426.

Marco Castellani

Department of "Sistemi ed Istituzioni per l'Economia",

University of L'Aquila,

Italy

Massimo Pappalardo

Department of "Matematica Applicata", University of Pisa,

Italy 